\title{
Spatial Angular Filtering by FSSs Made of Chains of Interconnected SRRs and CSRRs
}

\author{
J. D. Ortiz, J. D. Baena, Member, IEEE, V. Losada, F. Medina, Fellow, IEEE, and J. L. Araque
}

\begin{abstract}
Two frequency selective surfaces (FSSs) made of an infinite set of parallel 1-D chains of interconnected split ring resonators (I-SRRs) and interconnected complementary split ring resonators (I-CSRRs) are studied. The main result was that the central frequencies of the stopband and passband can be strongly tuned by controlling the angle of incidence.
\end{abstract}

Index Terms-Babinet's principle, frequency selective surface (FSS), spatial angular filter.

\section{INTRODUCTION}

$\mathbf{S}$ INCE decades ago, many researchers have been involved in the design of Frequency Selective Surfaces (FSS) [1], [2]. In more recent years, so-called metasurfaces have attracted much attention. By definition, a metasurface is just an FSS based on the unit cell of some previously proposed 3-D metamaterial. These unit cells are designed to be much smaller than the wavelength, thus avoiding grating lobes. Fig. 1(a) and (b) show two examples based on the use of split ring resonators (SRR) and complementary split ring resonators (CSRR). It is well known that the band-stop filtering behavior is provided by the sample based on SRRs while band-pass filtering features are obtained from CSRRs [3]. It has been also reported that those behaviors remain stable under oblique incidence [4]. The aim of this letter is to investigate the effects of connecting these resonators forming arrays of 1-D chains as shown in Fig. 1(c) and (d). We will demonstrate that the new structures provide angle-dependent tunability, in the sense that the central frequency of the bands can be significantly shifted by changing the angle of incidence. At a fixed frequency and for variable angle of incidence, this structure can filter directions of the incident radiation. Angular filtering of plane waves has been used for decades to increase the directivity of antennas and/or suppress sidelobes [5],

Manuscript received December 04, 2012; revised March 24, 2013 and July 01, 2013; accepted July 10, 2013. Date of publication August 07, 2013; date of current version August 30, 2013. This was supported by the Colombian Administrative Departament of Science, Technology and Innovation (COLCIENCIAS, project 1101-521-29389) and Spanish Ministerio de Ciencia e Innovación (project Consolider EMET CSD2008-00066 and TEC2010-16948).

J. D. Ortiz and J. D. Baena are with the Department of Physics, Universidad Nacional de Colombia, Bogotá, Colombia (e-mail: jdortizc@unal.edu.co; jdbaenad@unal.edu.co).

V. Losada is with the Department of Applied Physics I, Universidad de Sevilla, Sevilla, Spain (e-mail: losada@us.es).

F. Medina is with the Department of Electronics and Electromagnetism, Universidad de Sevilla, Sevilla, Spain (e-mail: medina@us.es).

J. L. Araque is with Electric and Electronics Engineering Department, Universidad Nacional de Colombia, Bogotá, Colombia (e-mail: jlaraqueq@unal. edu.co).

Color versions of one or more of the figures in this letter are available online at http://ieeexplore.ieee.org.

Digital Object Identifier 10.1109/LMWC.2013.2274997

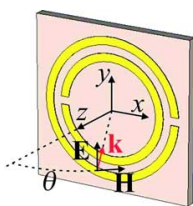

(a)

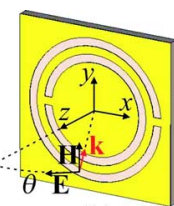

(b)

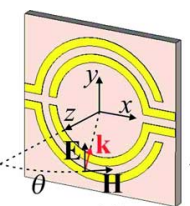

(c)

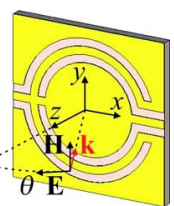

(d)
Fig. 1. Unit cells of metasurfaces made of unconnected SRRs (a), unconnected CSRRs (b), interconnected SRRs (I-SRRs) (c), and interconnected CSRRs (I-CSRRs) (d). Yellow color stands for copper and grey color for the dielectric substrate.

[6]. The structure described in this letter is advantageous when compared with previous implementations because of its significantly smaller electrical thickness.

\section{THEORY}

Since the introduction of the SRR [7], this structure has been modelled as an RLC circuit. Here we use the approximations for the self-inductance $\left(L_{\mathrm{SRR}}\right)$ and capacitance $\left(C_{\mathrm{SRR}}\right)$ of the SRR reported in [8, pp. 2574, 2580]. However the model for the unit cells depicted in Fig. 1(c) and (d) must be quite different due to the presence of the coplanar strip lines (or coplanar slots) connecting the chains of SRRs (or CSRRs). Each of the new unit cells will be called interconected SRR (I-SRR) and interconnected CSRR (I-CSRR). Let us now focus the attention on the drawing in Fig. 2(a) and the equivalent circuit model below [Fig. 2(b)]. The ring drawn with solid lines represents one unit cell (i.e., a single I-SRR), whereas the rings in dashed lines are its closest neighbors. We assume that the chain is periodical and therefore infinite. First, note that each original SRR has been cut just at the point where the electric current is supposed to have a maximum value for the fundamental resonance. In this point, a pair of coplanar strips has been included in order to transfer electric current between the neighboring cells. If the phase shift between the currents at the left and right transmission line sections is not important, the resonance of the I-SRR should be quite similar to that of the unconnected SRRs, which is the situation under normal incidence. However, for general oblique incidence, there is some phase shift between the input and output ports of the unit cell and surface waves arise rather than the resonance of each single resonator.

Assuming the unit cell is electrically short, a simplified lumped circuit model can be used to account for the electromagnetic behavior of the structure. The inductance and capacitance per unit length of the pair of coupled strips can be easily retrieved from the design formulas of characteristic impedance and phase constant in [9, pp. 43-44]. The inductance and the capacitance of the interconnection line section of length $l$ can be then approximated as $L_{\text {line }}=l L_{\mathrm{pul}}$ and $C_{\text {line }}=l C_{\mathrm{pul}}$. Then, by applying the Kirchhoff's circuit laws for the periodical 


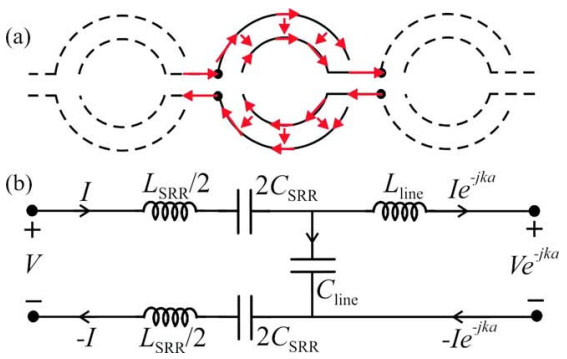

Fig. 2. (a) Sketch of a chain of interconnected SRRs. Red arrows represent electric and displacement currents. (b) Circuit model of the unit cell.

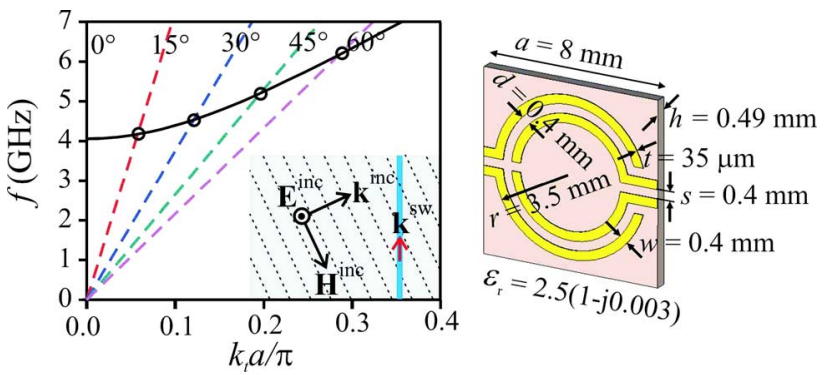

Fig. 3. Dispersion relation for a periodical chain of I-SRRs (solid line) and external incident waves with different angles of incidence (dashed lines).

circuit cell shown in Fig. 2(b), it is easy to get the dispersion relation for the periodic 1-D chain of I-SRRs

$$
\left(\frac{\omega}{\omega_{0}}\right)^{2}=\left(1+\frac{L_{\text {line }}}{L_{\mathrm{SRR}}}\right)^{-1}\left[1+2 \frac{C_{\mathrm{SRR}}}{C_{\text {line }}}(1-\cos (k a))\right]
$$

where $\omega_{0}=1 / \sqrt{L_{\mathrm{SRR}} C_{\mathrm{SRR}}}$ is the resonance frequency of the single SRR. If the coupling between two adjacent chains of resonators is negligible, the dispersion relation for surface waves propagating along the direction of the chains should be very similar to the expression (1). Fig. 3 shows the dispersion relation (solid line) predicted by (1) using the unit cell parameters therein. If we now consider an incident plane wave impinging upon the surface, it is expected that this wave will strongly couple to that surface wave whose wavevector exactly matches the transverse component of the incident wavevector. This idea is sketched in the inset of Fig. 3. Color dashed lines of Fig. 3 represent frequency versus tangential component of the incident wavevector for different incident angles. Therefore, the transmission dip (peak) of the metasurface of I-SRRs (I-CSRRs) should appear just at the frequency where the solid line intersects the dashed line. An increment of the angle of incidence means a decrease of the slope of the dashed line, thus provoking a shift to a higher resonance frequency.

\section{NUMERICAL SimULATIONS}

Simulations of the four geometries shown in Fig. 1 with parameters indicated in Fig. 3 were carried out by using the commercial simulator CST Microwave Studio. The transmission coefficients are shown in Fig. 4. For the cases of structures made of SRRs or I-SRRs, a stopband was always obtained while passbands are found for CSRRs or I-CSRRs. This is clearly in agreement with the Babinet's principle [1], [10]. It is noticeable that when the resonators are interconnected (I-SRRs or I-CSRRs) the central frequency depends on the angle of incidence. This is

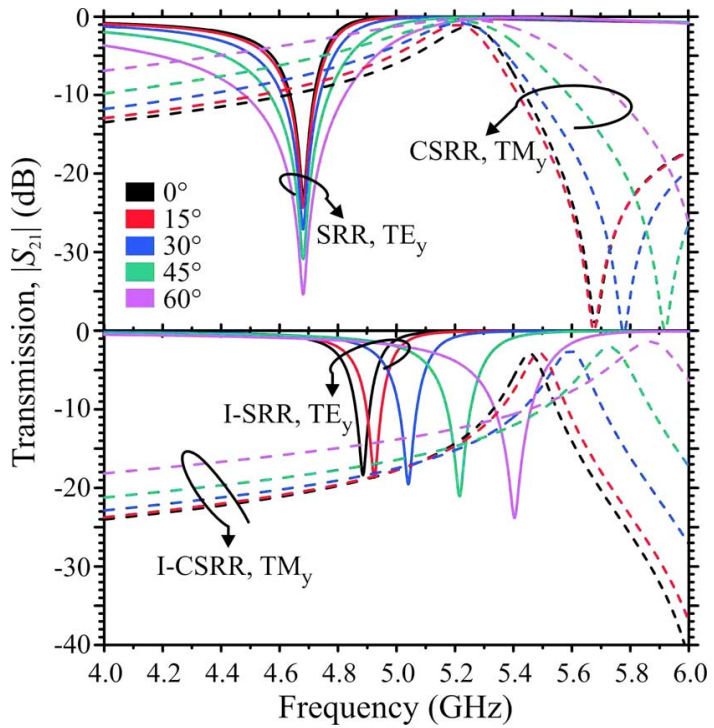

Fig. 4. Simulated transmission coefficients for metasurfaces made of unconnected resonators (up) and interconnected resonators (down).

not the case for unconnected resonators (SRRs and CSRRs). If the angle of incidence is swept from $0^{\circ}$ to $60^{\circ}$, the central frequency of the band shifts about $0.5 \mathrm{GHz}$, which means a $10 \%$ tunning. There is good qualitative agreement with the approximated theoretical model, in the sense that it predicts the shift to upper frequencies. There seems to be, however, a limitation of the theory presented: around $f=5 \mathrm{GHz}$ the wavelength is about $\lambda=60 \mathrm{~mm}$, that is 7.5 times the size of the unit cell $(8 \mathrm{~mm})$ what migth lead us to reject the hypothesis of an electrically small unit cell. To see why this is not the case, we note that what actually matters is whether the unit cell is much smaller than the effective transversal wavelength or not, being the latter $\lambda_{t}=\lambda / \sin \theta$. It is easy to find out that, in order to keep the unit cell smaller than $1 / 10$ the effective transversal wavelength, the angle of incidence should be less than $49^{\circ}$.

In order to demonstrate the mentioned application as spatial angular filter, we have depicted in Fig. 5 the transmission coefficient versus the angle of incidence at different fixed frequencies. For comparison purpose we add the response of a half-wavelength cavity shown in [5, Fig. 4]. It is clear that a single sheet of I-CSRRs behaves worse than the Kinowski's cavity. However, if we use two cascaded surfaces separated by $8 \mathrm{~mm}$, roughly $1 / 7$ the resonance wavelength, we get a very similar response to Kinowski's result (blue lines). Therefore, the main advantage of our proposal is the smaller electrical thickness.

\section{EXPERIMENT}

A first proof of concept experiment has been carried out (see Fig. 6) . A square sample with side length of $31.2 \mathrm{~cm}$ was printed on a commercial microwave substrate (ARLON CuClad $250 L X)$. A complete description of the sample and the experimental setup is given in Fig. 6. The characterization was done in anechoic environment by measuring the scattering parameter $S_{21}$ between two horn antennas placed far enough, in such a way that the incident wave was, approximately, a plane wave. In order to reduce edge diffraction, the sample was surrounded by several pieces of absorbing material. The sample was mounted 


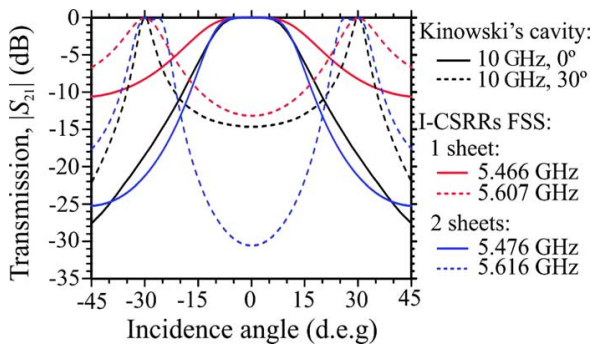

Fig. 5. Simulated transmission coefficients through Kinowski's cavity studied in [5] (black lines), one sheet of I-CSRRs (red lines), and two sheets of I-CSRRs separated by $8 \mathrm{~mm}$ (blue lines). Lossless materials are considered for direct comparison.
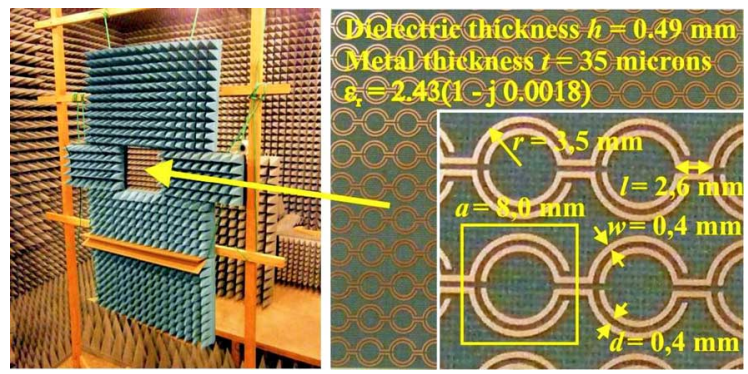

Fig. 6. Experimental setup. The sample is placed at the hole in the absorber wall. Lateral dimensions of the sample are $31.2 \times 31.2 \mathrm{~cm}^{2}$. Two horn antennas are placed in front and back of the FSS at a distance of $185 \mathrm{~cm}$.

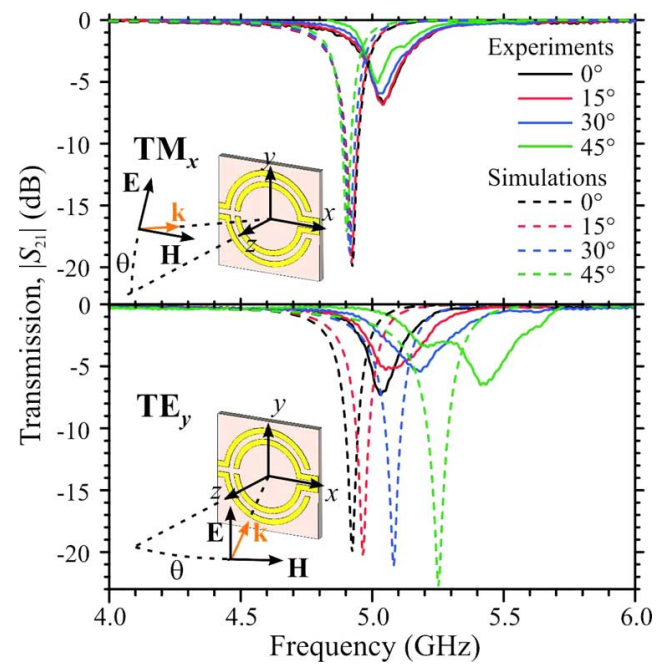

Fig. 7. Measured (solid lines) and simulated (dashed lines) transmission coefficients for polarizations $\mathrm{TM}_{x}$ and $\mathrm{TE}_{y}$ for different angles of incidence.

on a vertical axis allowing for rotation. A normalization was always done by taking as reference the transmission through the empty hole on the absorber. Fig. 7 shows the normalized transmission coefficients through the metasurfaces of I-SRRs for different angles of incidence. Fig. 7(a) shows that for $\mathrm{TM}_{x}$ incidence the resonance frequency remains invariant with respect to angle variations. This is due to the fact that the tangential component of the incident wave vector is orthogonal to the chains of connected resonators, in such a way that the surface wave cannot be excited. In order to observe a shift of the resonance frequency, the wave vector must have a varying projection along the direction of chains of resonators. Fig. 7(b) shows the measured transmission for polarization $\mathrm{TE}_{y}$. Now the shift is clearly observed. It is worth to note the qualitative agreement between the experimental (solid lines) and the simulation (dashed lines) results. The disagreement on the resonance depth is due to the fact that the chains of resonators are finite and thus cells near the edges can allow some level of transmission at the frequency at which they should strongly reject.

\section{CONCLUSION}

It has been demonstrated that some designs of metasurfaces made of interconnected resonators have the capability of providing spatial angular filtering. We started from old previous designs of filters using the split ring resonator (SRR) and its complementary structure (CSRR) as unit cells. The new idea was to connect them along one direction by using short pieces of transmission lines. The metasurfaces made of interconnected I-SRRs and I-CSRRs provide a stopband and a passband, respectively, whose central frequencies can be tuned by changing the angle of incidence without affecting too much to the bandwidth. These structures could be used as spatial angular filters for improving the directivity of an antenna or beam scanning by controlling the emission frequency.

\section{REFERENCES}

[1] B. A. Munk, Frequency Selective Surfaces: Theory and Design. New York: Wiley, 2000.

[2] T. K. Wu, Frequency Selective Surfaces and Grid Arrays. New York: Wiley, 1995

[3] F. Falcone, T. Lopetegi, M. A. G. Laso, J. D. Baena, J. Bonache, M. Beruete, R. Marqués, F. Martín, and M. Sorolla, "Babinet principle applied to the design of metasurfaces and metamaterials," Phys. Rev. Lett., vol. 93, p. 197401, 2004.

[4] M. Beruete, M. Sorolla, R. Marqués, J. D. Baena, and M. Freire, "Resonance and cross-polarization effects in conventional and complementary split ring resonator periodic screens," Electromagnetics, vol. 26, p. 247,2006

[5] D. Kinowski, M. Guglielmi, and A. G. Roederer, "Angular bandpass filters: An alternative viewpoint gives improved design flexibility," IEEE Trans. Antennas Propag., vol. 43, pp. 390-395, 1995.

[6] Y. J. Lee, J. Yeo, R. Mittra, and W. S. Park, "Application of electromagnetic bandgap (EBG) superstrates with controllable defects for a class of patch antennas as spatial angular filters," IEEE Trans. Microw. Theory Tech., vol. 53, pp. 224-235, 2005.

[7] J. B. Pendry, A. J. Holden, D. J. Robins, and W. J. Stewart, "Magnetism from conductors and enhanced nonlinear phenomena," IEEE Trans. Microw. Theory Tech., vol. 47, p. 2075, 1999.

[8] R. Marqués, F. Mesa, J. Martel, and F. Medina, "Comparative analysis of edge- and broadside-coupled split ring resonators for metamaterial design - theory and experiments," IEEE Trans. Antennas Propag., vol. 51, pp. 2572-2581, 2003.

[9] I. Bahl and P. Bhartia, Microwave Solid State Circuit Design. New York: Willey, 1988.

[10] J. D. Jackson, Classical Electrodynamics, 3rd ed. New York: Wiley, 1999. 